\title{
Mucoadhesive Tablets for Controlled Release of Acyclovir
}

\author{
Roberto Ruiz-Caro, ${ }^{a}$ Manuel Gago-Guillan, ${ }^{b}$ Francisco Javier Otero-Espinar, ${ }^{b}$ and \\ María Dolores Veiga*,a,c \\ ${ }^{a}$ Departamento de Farmacia y Tecnología Farmacéutica, Facultad de Farmacia, Universidad Complutense de \\ Madrid; Plaza Ramón y Cajal s/n, 28040-Madrid, Spain: ${ }^{b}$ Departamento de Farmacia y Tecnología Farmacéutica, \\ Facultad de Farmacia, Campus Universitario sur s/n; 15782-Santiago de Compostela, Spain: and ${ }^{c}$ Unidad de \\ Biotransformaciones Industriales; Parque Cientifico de Madrid, PTM, C/Santiago Grisolía 2, 28760_Tres Cantos, \\ Madrid, Spain.
}

Received April 10, 2012; accepted July 17, 2012; advance publication released online August 2, 2012

\begin{abstract}
Mucoadhesive chitosan (CS) and/or hydroxypropyl-methylcellulose (HPMC) tablets for gastric drug delivery of acyclovir (ACV) have been developed in order to improve the ACV oral bioavailability. Swelling, bioadhesive and dissolution studies were carried out in two acidic media (pH 1.5 and 4 ) in order to determine the tablets behaviour in both fed and fasted states. All the designed tablets showed good mucoadhesive properties on gastric mucosa due to the presence of CS and/or HPMC. In vitro dissolution of ACV from tablets was influenced by the swelling behaviour of each polymer. All data release of the studied tablets fitted to Hopfenberg model, which describes drug release from tablets displaying heterogeneous erosion. HPMC and CS/HPMC tablets revealed a sustained release for $24 \mathrm{~h}$, but a complete dissolution of the tablets was not produced at this time. On the contrary, tablets which contained only CS as polymer were able to release the total amount of $\mathrm{ACV}$ for $4 \mathrm{~h}$, due to the $\mathrm{CS}$ imbibition and erosion processes in pH $1.5 \mathrm{medium}$. These results allowed us to conclude that CS is the excipient to be chosen to obtain gastroretentive formulations, due to its demonstrated gastric compatibility.
\end{abstract}

Key words acyclovir; chitosan; hydroxypropyl-methylcellulose; mucoadhesivity; drug controlled release; swelling behaviour

In recent years, the excipients choice for drug formulation has focused on natural and biocompatible products. Chitosan (CS) is a biocompatible material obtained from an abundant natural polysaccharide like chitin by deacetylation processes. ${ }^{1)}$ Several excellent reviews have compiled different methods for obtaining a wide range of chitosans with different deacetylation degrees and molecular weights which affect some of their physico-chemical parameters. ${ }^{2-4)}$ In pharmaceutical technology field CS has found applicability as a potential formulation excipient acting as a disintegrant, binder or tablet coating agent. This polymer presents a swelling ability when it is placed within an aqueous media, ${ }^{5)}$ and mucoadhesivity in the oral cavity ${ }^{6-8)}$ and in the gastrointestinal tract. ${ }^{9)} \mathrm{CS}$ is a well-known polycation that reacts with negatively charged components, either ions or molecules, leading to the formation of polyelectrolyte complex through ionic bridges between polymer chains. Their mucoadhesive property is mainly based on the ionic interaction with anionic substructures of mucous layer. $^{10)}$

Hydroxypropyl-methylcellulose (HPMC) is a non-ionic cellulose ether which has methoxylic and hydroxypropoxylic moieties substituted onto the glucose units. Types of HPMC differ by various degrees of substitution of hydroxypropyl (hydrophilic) and methoxy (hydrophobic) groups and hence its versatility to retard drug release of various solubilities due to the ratio of hydroxypropyl to methoxyl content affects the extent of polymer interaction with water. ${ }^{11)}$ This cellulose ether is the hydrophilic and swellable polymer most commonly used for the preparation of oral controlled drug delivery systems. ${ }^{12-14)}$ Upon contact with water or biological fluid, drug release from HPMC matrices is controlled by hydration of the

The authors declare no conflict of interest. polymer, which forms a gelatinous barrier layer at the surface of the tablet through which the drug diffuses. First, the system absorbs rapidly water by diffusion and the volume expansion of the matrix forms a gel layer around the central, solid and dry inner core (polymer chain relaxation). Finally, drug release in soluble drugs is mainly controlled by drug diffusion through the gel layer whereas release for poorly water-soluble drugs, is likely to be controlled by a combination of diffusion and gel layer erosion (relaxation-dissolution polymer). ${ }^{15-19)}$

Treatments against human herpes simplex virus type-1 (HSV-1) are composed of a nucleoside analogue that inhibits herpes virus DNA polymerase, such as acyclovir (ACV) or valacyclovir. ${ }^{20)} \mathrm{ACV}$ is absorbed paracellularly by passive diffusion from the upper region to the duodenum or jejunum regions. ${ }^{21,22)}$ However, some reports have suggested that the high variability of ACV effectiveness is related to saturable and dose dependent processes, ${ }^{23)}$ thus its bioavailability is reduced to $15-30 \%{ }^{24)}$ due to short residence time at the absorption site. Instead, the inclusion of absorption-enhancing excipients in formulations can enhance ACV bioavailability. So, Attia et al. developed ACV niosomes composed by cholesterol, Span 60, and dicetyl phosphate to improve its oral bioavailability with positive results in rabbits. ${ }^{25}$ )

Some strategies to improve the absorption of different drugs from the gastrointestinal tract are based on the use of mucoadhesive delivery systems. Mucoadhesion is a complex phenomenon. There are different theories that might explain the mechanism of mucoadhesion occurred between bioadhesive polymers and biological mucosal surface, including electronic, wetting, adsorption, diffusion, mechanical and fracture theories. $^{26)}$ The occurrence of mucoadhesion has been said to experience two stages, the contact (wetting) stage followed by the consolidation stage (the establishment of adhesive 
interactions). ${ }^{27)}$ However, the in vivo mucoadhesion is influenced by many factors, such as various mucus turnover time, $\mathrm{pH}$, condition, and composition of different mucus. It is critical to employ an appropriate in vitro model to help to evaluate the mucoadhesiveness and predict in vivo behaviour of the formulation. ${ }^{28)}$ So, Belgamwar et al. prepared mucoadhesive multiparticulate system of metoprolol and atenolol composed of various mucoadhesive polymers including HPMC of various grades like K4M, K15M, K100M, E50LV, Carbopol of grades 971P, 974P and polycarbophil for oral drug delivery which was retarded crosslinking sodium alginate with calcium chloride. The prepared formulations exhibited good mucoadhesive strength and a good swelling index; however, the mucoadhesive studies showed that HPMC had greater mucoadhesive properties than Carbopol and polycarbophil. Metoprolol and atenolol release from the multiparticulate system that was regulated and extended until $12 \mathrm{~h}$ exhibiting non-Fickian drug release kinetics. ${ }^{29)}$

In the case of $\mathrm{ACV}$, different studies about the development of gastroadhesive dosage forms have been carried out. In this sense, Dhaliwal et al. evaluated in vitro and in vivo mucoadhesive microspheres of ACV where CS, thiolated CS, Carbopol $71 \mathrm{G}$ and Methocel K15M were used as mucoadhesive polymers obtaining a prolonged release of drug during $12 \mathrm{~h}$ with a significant improvement in oral bioavailability of ACV due to the increase in the retention in the upper gastrointestinal tract. ${ }^{30)}$ On the other hand different ratios of Ethylcellulose/Carbopol have been used to prepare ACV mucoadhesive microspheres which could remain in the stomach for an extended time and help ACV to be better absorbed in the small intestinal tract. ${ }^{28)}$ More recently calcium alginate or resinates have been chosen as excipients to develop ACV microspheres with gastroadhesivity and controlled drug release in intestinal zone. ${ }^{31,32)}$ Nevertheless, there are not studies about the development of gastroadhesive solid dosage forms, like tablets, for ACV controlled release.

With this background the aim of this research is to develop modulated gastric delivery monolithic systems (tablets) based on mucoadhesive polymers, such as CS or HPMC, for poorly absorbed orally administered drugs. ACV was chosen as model drug being poorly absorbed from the gastrointestinal tract in an attempt to increase ACV bioavailability. These tablets should display strong mucoadhesive properties and a sustained drug release. The development and in vitro evaluation of a delivery system meeting these demands is described within this study.

\section{Experimental}

Materials ACV (batch 14070620002) was a generous gift from CINFA (Pamplona, Spain). CS (batch 8826900003) with a kinematic viscosity of $92.0 \times 10^{-6} \mathrm{~m}^{2} / \mathrm{s}$ and a deacetylation degree of $97.0 \%$ was purchased from Guinama (Valencia, Spain). HPMC-Methoce ${ }^{\circledR}$ K15M CR PREMIUM (batch QJ19012N11) with a viscosity grade of $15000 \mathrm{cPs}$ as a $2 \% \mathrm{w} / \mathrm{v}$ solution in water at $20^{\circ} \mathrm{C}$ was manufactured by Dow Chemical Company and supplied by Colorcon (Kent, U.K.). The diluent used for manufacturing the tablets studied in this paper was anhydrous dibasic calcium phosphate "Anhydrous Emcompress $^{\circledR}$ " (ADP) (batch 1006) being purchased from Mendell (Bodenheim, Germany). Magnesium stearate PRS-CODEX ${ }^{\circledR}$ (MgSt) (batch 028) was provided by Panreac (Barcelona, Spain) and Kollidon ${ }^{\circledR} 30$ (PVP K30) (batch 98-0820) was supplied by BASF (Ludwingshafen, Germany). All other reagents were of analytical grade. The water used was demineralized in all cases.

Preparation of ACV Matrix Tablets Five different batches of matrix tablets containing $100 \mathrm{mg}$ of ACV have been prepared using wet granulation. First, all necessary components were previously sieved through a mesh size of $100 \mu \mathrm{m}$. In every case, the ingredients included in the Table 1 (ACV, HPMC, CS and/or ADP) were mixed homogeneously. A known quantity of PVP was dissolved in the enough amount of ethanol for achieving a damp mass and this solution was gradually added and mixed continuously to the previous mix to form a damp mass, that was forced through a mesh size of $0.5 \mathrm{~mm}$ and the granules obtained were dried at $40^{\circ} \mathrm{C}$ for $12 \mathrm{~h}$. Dried granules were lubricated with $1 \% \operatorname{MgSt}(\mathrm{w} / \mathrm{w})$ and tablets with an average weight of $400 \mathrm{mg}$ and a diameter of $12 \mathrm{~mm}$ were obtained compressing the lubricated granules using an eccentric machine Bonals ${ }^{\circledR}$ B 40 (Barcelona, Spain), fitted with a $12 \mathrm{~mm}$ diameter flat and circular punch and applying the maximum compression force accepted by the formulation. The compaction pressure was kept constant during the preparation of every batch. The obtained tablets have satisfied the friability tests, average weight, drug content, and diameter and thickness studies. The composition of CS, HPMC, ADP and ACV in every tablet of all batches prepared is shown in Table 1.

Characterization of ACV Mucoadhesive Matrix Tablets. Swelling Test The gastric $\mathrm{pH}$ in fasted subjects is 1.1. $0.15^{33-35)}$ and in fed state is 2.0 to $6.0,{ }^{36)}$ for this reason, the swelling, mucoadhesivity and dissolution experiments were carried out in two acid media ( $\mathrm{pH} 1.5$ or 4). The first medium was composed of an aqueous mixture of $0.05 \mathrm{M}$ hydrochloric acid $37 \%, 0.05 \mathrm{~m}$ ortho-phosphoric acid $85 \%$ and $0.05 \mathrm{~m}$ acetic acid glacial with a $\mathrm{pH}$ value of 1.5 . The second medium was prepared with the same aqueous mixture of acids however a sufficient quantity of $\mathrm{NaOH} 10 \mathrm{~m}$ was added until $\mathrm{pH}$ reached a value of 4.0.

Swelling behaviour of systems has been carried out in order to determine their influence on $\mathrm{ACV}$ controlled release from batches $\mathrm{A} \rightarrow \mathrm{E}$. Swelling ratio was evaluated as weight gain

Table 1. Composition of the Prepared Mucoadhesive Acyclovir Tablets

\begin{tabular}{ccccc}
\hline \hline Batch & CS (mg) & HPMC (mg) & ADP (mg) & ACV (mg) \\
\hline A & 135 & - & 135 & 100 \\
B & 180 & - & 70 & 100 \\
C & 200 & 135 & 135 & 100 \\
D & - & 135 & - & 100 \\
E & 135 & & & 100 \\
\hline
\end{tabular}




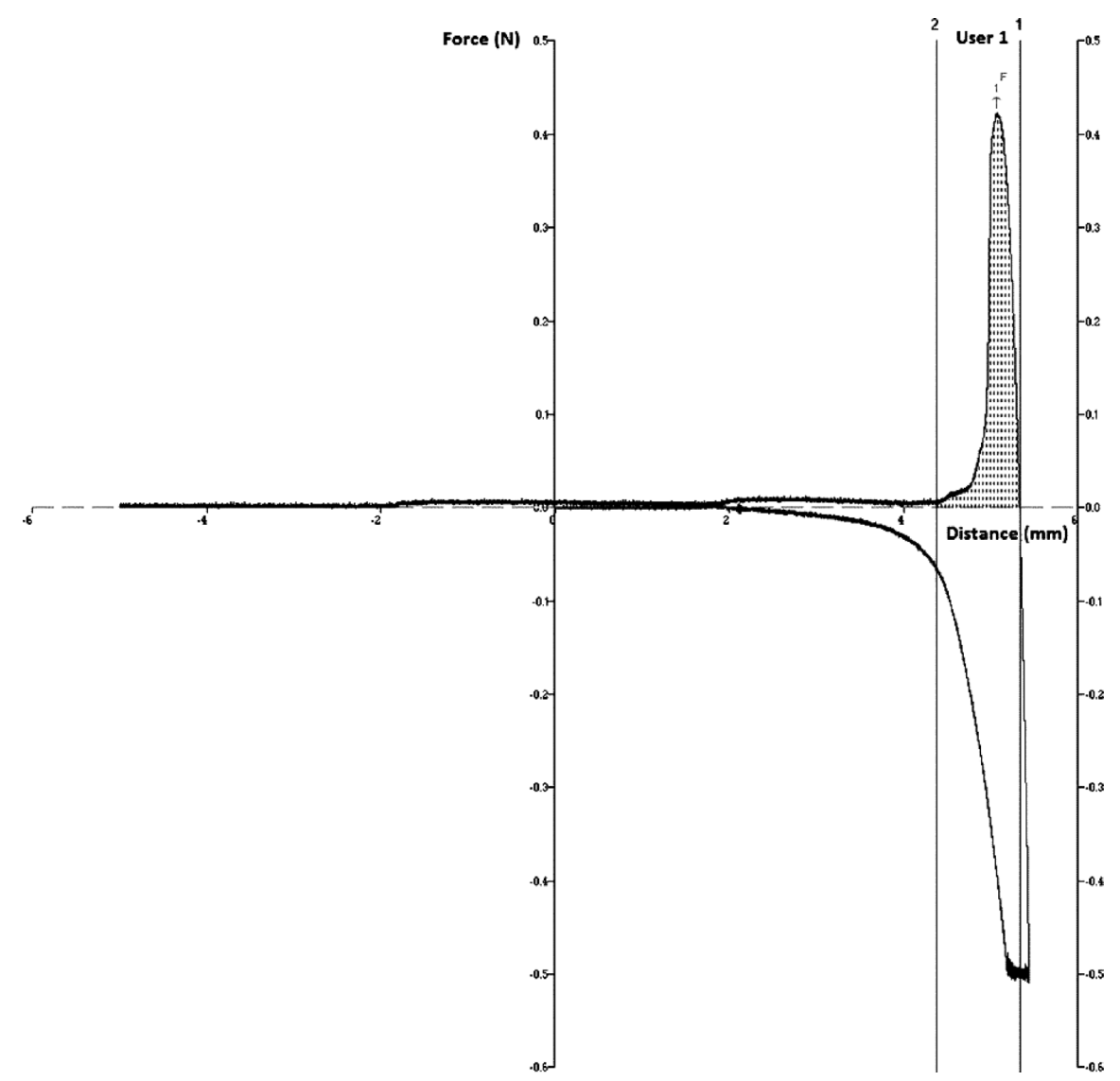

Fig. 1. Example of Force versus Elongation Graph Registered at $\mathrm{pH} 1.5$ for One Replicate of the Batch 5

in both media described above, at a medium temperature of $37 \pm 0.1^{\circ} \mathrm{C}$. The maximum duration of the tests was $24 \mathrm{~h}$ in the batches D and E (tablets with HPMC) and $6 \mathrm{~h}$ in batches $\mathrm{A}, \mathrm{B}$ and $\mathrm{C}$ (tablets without HPMC). Before the introduction of the systems in the test medium, each tablet was fixed to a metallic disc of $20 \mathrm{~mm}$ using a cyanoacrylate adhesive (Loctite $^{\circledR}$, Henkel, Austria) in order to keep constant the contact of tablet surface with the aqueous medium and to improve the handling of the tablets during the test. At specific time intervals, the samples were removed from the test medium with a magnetic rod and were blotted with filter paper to absorb the excess of liquid on sample surface. At $t=30,180,360,480$ and $1440 \mathrm{~min}$, photographs were taken with a digital camera (Panasonic $^{\circledR}$ Lumix DMC-FZ28 10.0 Megapixels, Osaka, Japan), to observe the appearance and evolution of tablets (batches B, D and E) in contact with the media. The swelling ratio (SR \%) of every sample was calculated according to the Eq. $1^{37)}$ :

$$
\mathrm{SR} \%=\left[\left(T_{\mathrm{s}}-T_{\mathrm{d}}\right) / T_{\mathrm{d}}\right] \times 100
$$

where $T_{\mathrm{s}}$ and $T_{\mathrm{d}}$ were the weights of the swollen and dried samples, respectively. All swelling tests were performed in triplicate.

In Vitro Evaluation of the Mucoadhesive Properties by Bioadhesion Studies Studies of mucoadhesivity of systems have been carried out to observe the in vitro adhesive behaviour of the tablets in a mucosa similar to the human in order to prolong the time in the stomach and therefore achieve a sustained release of ACV.

For substrate preparation, samples of stomach porcine mucosa were obtained from a local slaughterhouse immediately after the animal had been sacrificed. The mucosa was carefully cleaned to eliminate food residues and separated from the muscular tissue. Mucosa was cut into pieces with suitable size for bioadhesion testing, and pieces were packed in individual containers and kept in the freezer. Before using, samples were defrosted in a fridge at temperatures between $0-4^{\circ} \mathrm{C}$.

Bioadhesive capacity of tablets was determined by measuring the maximum detachment force and the bioadhesion work using a TA-XT2i Texture Analyzer (Stable Micro Systems Ltd., Surrey, U.K.) fitted with a constant temperature chamber. Conditions of the work were established on the basis of previous studies. ${ }^{38,39)}$ The tablet was stuck to the upper support and the stomach mucosa to Petri dishes by using a cyanoacrylate adhesive (Loctite, Henkel, Austria). Petri dishes were then attached to the lower support. The assay was performed at $\mathrm{pH} 1.5$ and $\mathrm{pH} 4.0$ using the buffers described for swelling tests. Mucosal tissue was covered with $15 \mathrm{~mL}$ of a pre-warmed fluid (immersion fluid) and keep until $37^{\circ} \mathrm{C}$ was stabilised. Afterwards, upper support was descended at $5 \mathrm{~mm} / \mathrm{min}$ upon contact of the tablet-substrate at a defined force of $0.5 \mathrm{~N}$. This force was maintained for $5 \mathrm{~min}$ to ensure the intimate contact between tablets and stomach mucosa. Finally, an extension phase was developed using a rate of $5 \mathrm{~mm} / \mathrm{min}$ until total separation of the components was achieved. During this experiment the force $(\mathrm{N})$ versus elongation was registered, and bioadhesion work was calculated $(\mathrm{mJ})$ as the area below the obtained graph in the final extension phase (see example in Fig. 1). All bioadhesion tests were performed in five replicate. 
Two-way analysis of variance (Two way ANOVA, Statgraphics ${ }^{\circledR}$ plus 5.1) was used to compare the bioadhesive behaviour of tablets after testing for normality and homogeneity of variance.

Dissolution Study A Sotax ${ }^{\circledR}$ AT-7 dissolution apparatus (Basel, Switzerland) with paddles was employed to carry out all tests. The paddle speed, experimental temperature and volume of the dissolution medium were $100 \mathrm{rpm}, 37 \pm 0.1^{\circ} \mathrm{C}$ and $900 \mathrm{~mL}$ of the two acid media ( $\mathrm{pH} 1.5$ and 4) described previously, respectively. The maximum duration of the tests was $24 \mathrm{~h}$ in the batches D and E (tablets with HPMC) and $6 \mathrm{~h}$ in A, B and C (tablets without HPMC). Samples were withdrawn at specific time intervals and filtered using Whatman ${ }^{\circledR}$ filter papers (type 42). The quantity of dissolved ACV was determined by UV spectroscopy at a wavelength of $255 \mathrm{~nm}(\mathrm{pH}$ $1.5)$ and at $251.5 \mathrm{~nm}(\mathrm{pH} 4)$, using in all cases a Beckman ${ }^{\circledR}$ DU-7 spectrophotometer (Brea, CA, U.S.A.). Three replicates of each dissolution test were carried out. No changes in the $\lambda_{\max }$ of ACV because of the presence of tablet excipients had previously been checked.

In order to investigate the mechanism of ACV release from tablets $\mathrm{A} \rightarrow \mathrm{E}$, the mean release data were analyzed according to the following mathematical models, First order $\left(Q_{\mathrm{t}} \leq 1.0\right)$ (Eq. 2), ${ }^{40)}$ Higuchi $\left(M_{\mathrm{t}} / M_{\infty} \leq 0.6\right)$ (Eq. 3), ${ }^{41)}$ Hixson-Crowell or cube root $\left(M_{\mathrm{t}} / M_{\infty}=1-W_{\mathrm{t}} / W_{0} \leq 1.0\right)$ (Eq. 4), ${ }^{42)}$ Korsmeyer and Peppas $\left(M_{\mathrm{t}} / M_{\infty} \leq 0.6\right)\left(\right.$ Eq. 5) ${ }^{43)}$ and Hopfenberg kinetic $\left(M_{\mathrm{t}} / M_{\infty} \leq 1.0\right)(\text { Eq. } 6)^{44)}$.

$$
\begin{gathered}
Q_{\mathrm{t}}=Q_{0} e^{-K_{\mathrm{tt}}} \\
M_{\mathrm{t}} / M_{\infty}=K_{\mathrm{h}} t^{1 / 2} \\
W_{0}^{1 / 3}-W_{\mathrm{t}}^{1 / 3}=K_{\mathrm{s}} t \\
M_{\mathrm{t}} / M_{\infty}=a t^{n_{1}} \\
M_{\mathrm{t}} / M_{\infty}=1-\left[1-K_{0} t / C_{0} a_{0}\right]^{n_{2}}
\end{gathered}
$$

where $Q_{\mathrm{t}}$ is the amount of drug dissolved in time $t, Q_{0}$ is the initial amount of drug in the solution (most times, $Q_{0}=0$ ), $K_{1}$ is the first order release constant, $M_{\mathrm{t}} / M_{\infty}$ is the fraction of drug released at time $t$ (the drug loading was considered as $M_{\infty}$ ), $K_{\mathrm{H}}$ is the Higuchi dissolution constant, $W_{0}$ is the initial amount of drug in the tablet, $W_{\mathrm{t}}$ is the remaining amount of drug in the tablet at time $t, K_{\mathrm{s}}$ is a constant incorporating the surfacevolume relation, $a$ is a constant incorporating structural and geometric characteristics of the drug dosage form, $n_{1}$ is the release exponent, indicative of the drug release mechanism, $K_{0}$ is the erosion rate constant, $C_{0}$ is the initial concentration of drug in the matrix and $a$ is the initial radius for a sphere or cylinder or the half-thickness for a slab. The value of $n_{2}$ is 1 , 2 and 3 for a slab, cylinder and sphere, respectively. For this equation, $K_{1}=K_{0} / C_{0} a_{0}$.

\section{Results and Discussion}

Swelling Test Figures 2 and 3 show the swelling ratios of systems in acidic medium $\mathrm{pH} 1.5$ and in acidic medium $\mathrm{pH} 4$ respectively, where each positive swelling ratio value indicates that, at this time, the swollen system weight was higher than the dry system weight $(t=0)$. On the other hand, each negative swelling ratio value indicated that the weight of the swollen

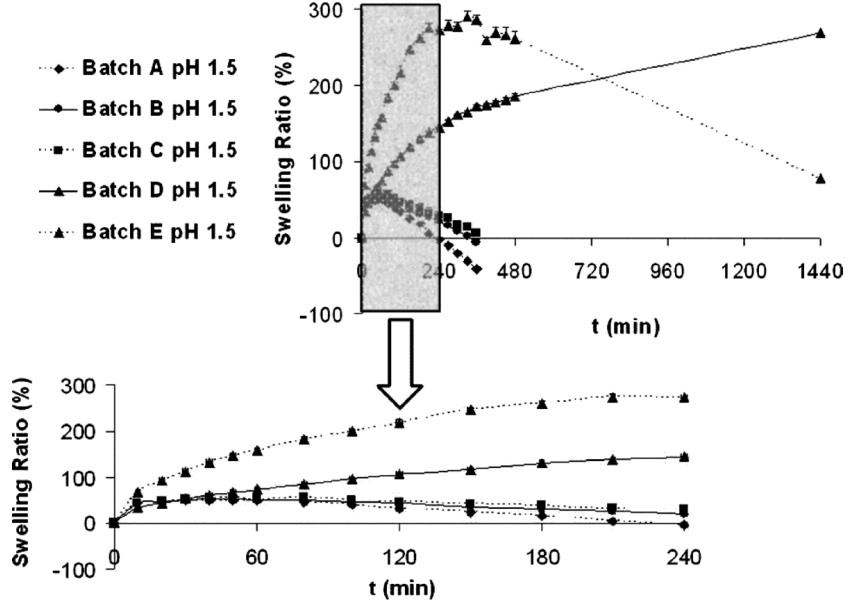

Fig. 2. Swelling Ratio of Tablets in Acidic Medium of pH 1.5 (Mean Values \pm Standard Deviation, $n=3$ )

-. Batch A pH 4

- Batch B pH 4

- Batch C pH 4

$\neg$ Batch D pH 4

.. Batch E pH 4
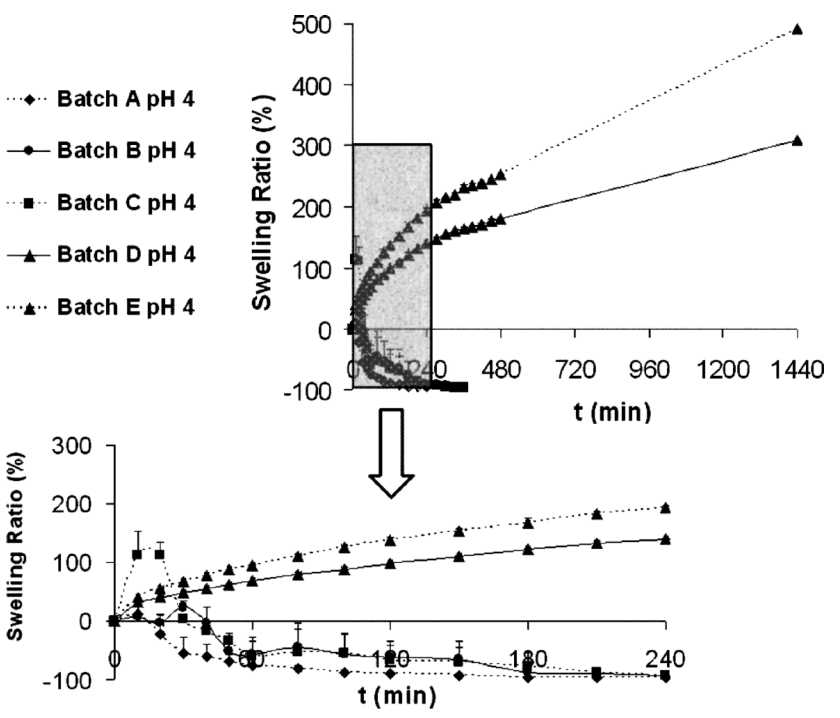

Fig. 3. Swelling Ratio of Tablets in Acidic Medium of $\mathrm{pH} 4$ (Mean Values \pm Standard Deviation, $n=3$ )

system was lower than the weight of the dry system $(t=0)$. When $t=0$, the swelling ratio value is 0 in all systems, due to the application of the equation shown in the experimental section.

Tablets with different proportions of CS (batches A, B and $\mathrm{C}$ ) showed similar swelling ratio evolution versus time. Their profiles reflected the characteristic behaviour in aqueous media of CS: imbibition and erosion. ${ }^{45)}$ For this reason, after $6 \mathrm{~h}$ of swelling test in both media ( $\mathrm{pH} 1.5$ and 4), only a few residues of each tablet remained fixed to the metallic discs (Fig. 4).

When CS was replaced by HPMC (batch D) tablets only displayed weight increase during all the time of the test, even after $24 \mathrm{~h}$ the tablets remained intact, showing no disintegration in both media although a weak transparent gel layer at $\mathrm{pH} 1.5$, in comparison with the corresponding at $\mathrm{pH} 4$, was observed at $1440 \mathrm{~min}$ (Fig. 4). This fact was due to the high imbibition capacity of HPMC, which created a gel layer around the tablet. In consequence, the imbibition of the tablet core was made impossible. 


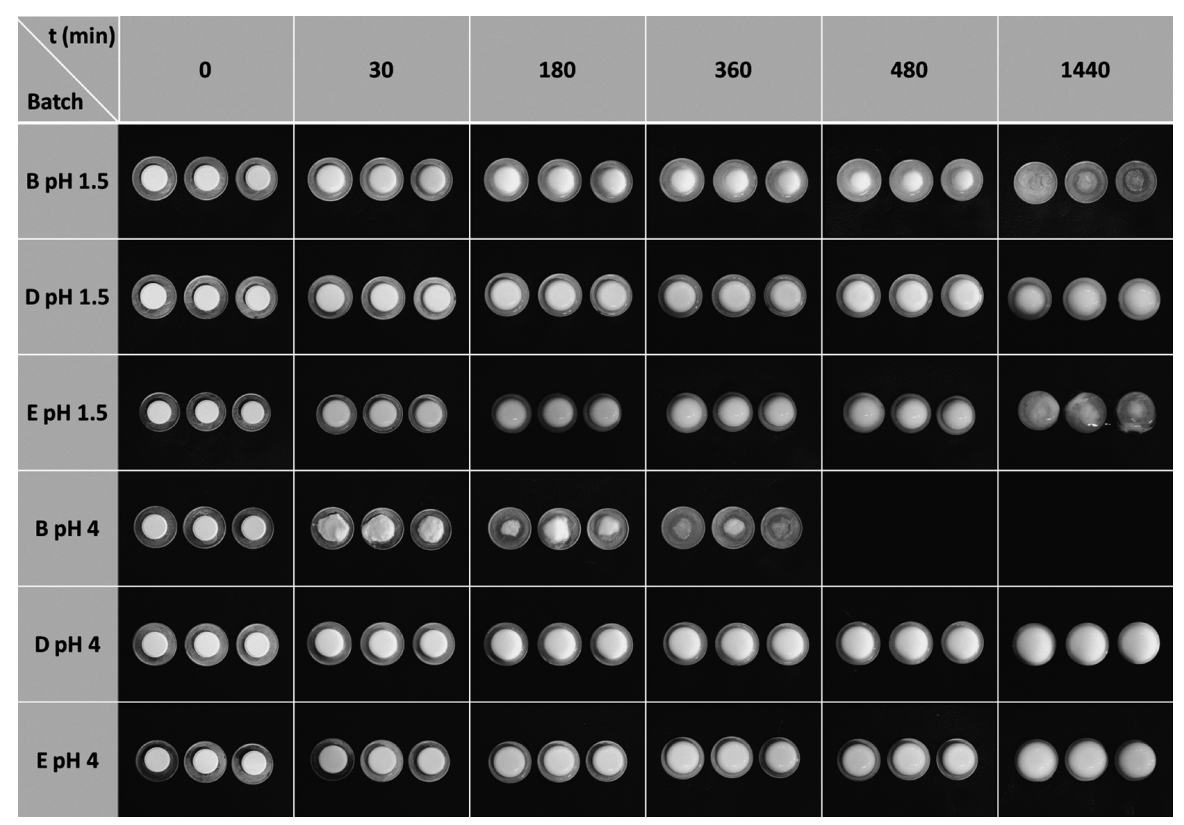

Fig. 4. Evolution of Batches B, D and E for Swelling Study in Both Acidic Media (pH 1.5 and pH 4)

In order to obtain an intermediate swelling behaviour, a batch with both polymers (batch E) was tested. The simultaneous presence of CS and HPMC in these tablets was the responsible of the surprising swelling behaviour in both media. The characteristic erosion of CS in acidic media was nullified by the presence of HPMC and even, higher amounts of aqueous media were embebed for these tablets in respect to the tablets with HPMC as only polymer (batch D), as can be seen in Fig. 4. However, after $8 \mathrm{~h}$ a decrease in the matrix weight was started, and after $24 \mathrm{~h}$ at $\mathrm{pH} 1.5$ only rests of tablet remained (Fig. 2). On the contrary, at $\mathrm{pH} 4$, due to the less solubility of HPMC at this $\mathrm{pH}$ value (batch D, Fig. 4), the gel layer continued growing after $24 \mathrm{~h}$ showing higher swelling ratio values (Fig. 3).

Bioadhesion Studies Table 2 shows bioadhesive properties in gastric conditions, expressed as bioadhesion work and maximum detachment force, for the different tablets assayed. As seen, all formulations showed good mucoadhesion behaviour on gastric mucosa.

Statistical analysis (Table 3) shows that the composition of tablets and the interaction between composition and medium $\mathrm{pH}$ are significant factors that influence both bioadhesion work and maximum detachment force. Nevertheless, $\mathrm{pH}$ was only significant in maximum detachment force but not in bioadhesive work.

The influence of the CS content on bioadhesive properties of tablets is showed in Fig. 5. It can be observed that at $\mathrm{pH} 1.5$ bioadhesion is practically independent for CS levels, nevertheless, in assays carried on at $\mathrm{pH} 4$ both bioadhesive work and maximum detachment force increases with proportion of CS.

These results are related with the cationic nature of the CS and the particular composition of the gastric mucin. In pig and in men, there are marked differences between the carbohydrate chains of the glycoproteins from the different regions of the gastrointestinal tract. The carbohydrate side chain of pig gastric mucus glycoprotein is complex, with a maximum chain length of 19 sugar residues and they have different amounts of negative charge on the carbohydrate side chains that in other intestinal regions. ${ }^{46)}$ Pig small-intestinal mucus and pig colonic mucus glycoprotein, are rich in both sialic acid and ester sulphate residues. ${ }^{47)}$ Is well know that the $\mathrm{p} K_{\mathrm{a}}$ of the sialic acid, also called $\mathrm{N}$-acetylneuraminic acid, is approximately 2.6 , so at intestinal $\mathrm{pH}(>4)$, glycoproteins are totally ionized and negatively charged producing the expansion of the mucus. In these conditions, gel mucus is more porous and electrostatic interactions may have importance in the bioadhesive forces. Nevertheless, similarly to the man, pig gastric mucus glycoprotein has an ester sulphate content that is equivalent to about one per 15 sugar residues together with a small amount of sialic acid. ${ }^{46)}$ At $\mathrm{pH} 1.5$ glycoproteins are practically unionised and at $\mathrm{pH} 4$ the sialic acid is ionised but due to their low proportion gastric mucosa have a significant low density

Table 2. Gastric Bioadhesive Behaviour of Acyclovir Tablets in Function of Their Composition (Means \pm Standard Deviation, $n=5$ )

\begin{tabular}{|c|c|c|c|c|}
\hline \multirow{2}{*}{ Batch } & \multicolumn{2}{|c|}{ Work of bioadhesion $(\mathrm{N} \cdot \mathrm{mm})$} & \multicolumn{2}{|c|}{ Maximum detachment force $(\mathrm{N})$} \\
\hline & $\mathrm{pH} 1.5$ & $\mathrm{pH} 4.0$ & $\mathrm{pH} 1.5$ & $\mathrm{pH} 4.0$ \\
\hline A & $0.07 \pm 0.04$ & $0.05 \pm 0.02$ & $0.10 \pm 0.02$ & $0.09 \pm 0.03$ \\
\hline $\mathrm{B}$ & $0.10 \pm 0.04$ & $0.07 \pm 0.02$ & $0.09 \pm 0.02$ & $0.26 \pm 0.09$ \\
\hline $\mathrm{C}$ & $0.05 \pm 0.02$ & $0.17 \pm 0.04$ & $0.08 \pm 0.02$ & $0.25 \pm 0.08$ \\
\hline $\mathrm{D}$ & $0.18 \pm 0.06$ & $0.21 \pm 0.05$ & $0.29 \pm 0.08$ & $0.25 \pm 0.03$ \\
\hline $\mathrm{E}$ & $0.14 \pm 0.03$ & $0.09 \pm 0.03$ & $0.43 \pm 0.10$ & $0.49 \pm 0.14$ \\
\hline
\end{tabular}

All tablets included $100 \mathrm{mg}$ of acyclovir, $27 \mathrm{mg}$ of PVP K30 and $3 \mathrm{mg}$ of magnesium stearate. 
Table 3. Two Way Analysis of Variance for the Parameters Work of Bioadhesion and Maximum Detachment Force Measured in Gastric Mucosa in Function of the Composition of Tablets and $\mathrm{pH}$ of Assay

\begin{tabular}{|c|c|c|c|c|c|c|}
\hline \multicolumn{2}{|l|}{ Source } & \multirow[t]{2}{*}{ Sum of squares } & \multirow[t]{2}{*}{$\mathrm{df}$} & \multirow[t]{2}{*}{ Mean squares } & \multirow[t]{2}{*}{$F$} & \multirow[t]{2}{*}{$\alpha$} \\
\hline Work of bioadhesion & Main effect & & & & & \\
\hline \multirow{11}{*}{$\begin{array}{l}\text { Maximum detachment } \\
\text { force }\end{array}$} & Batch & 0.1047060 & 4 & 0.02617660 & 20.64 & $<0.01$ \\
\hline & $\mathrm{pH}$ & 0.0017405 & 1 & 0.00174050 & 1.37 & ns \\
\hline & Interactions & 0.0465074 & 4 & 0.01162690 & 9.17 & $<0.01$ \\
\hline & Within & 0.0507204 & 40 & 0.00126801 & & \\
\hline & Total & 0.2036740 & 49 & & & \\
\hline & Main effect & & & & & \\
\hline & Batch & 0.8075270 & 4 & 0.20188200 & 38.98 & $<0.01$ \\
\hline & $\mathrm{pH}$ & 0.0563137 & 1 & 0.05631370 & 10.87 & $<0.01$ \\
\hline & Interactions & 0.1001470 & 4 & 0.02503680 & 4.83 & $<0.01$ \\
\hline & Within & 0.2071740 & 40 & 0.00517936 & & \\
\hline & Total & 1.1711600 & 49 & & & \\
\hline
\end{tabular}
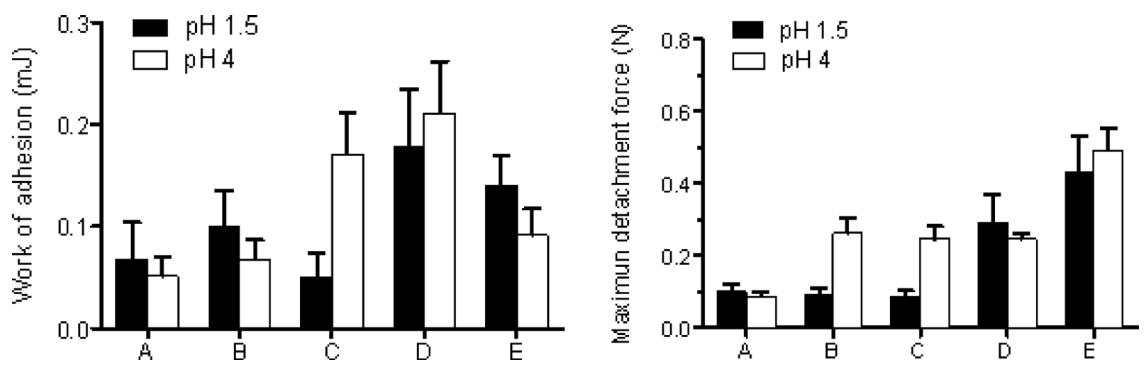

Fig. 5. Gastric Bioadhesive Behaviour of Acyclovir Tablets Elaborates without HPMC in Function of Chitosan Content and Comparison of Bioadhesive Properties of Acyclovir Tablets in Function of Their Composition (Mean Values \pm Standard Deviation, $n=5$ )

of charges and there is more compact (less porous gel) that intestinal mucosa. Consequently, at this level electrostatic interactions have less importance in the establishment of the bioadhesive forces and the secondary chemical bound (van der Waals forces and hydrogen and hydrophobic bonds) takes relevance. So, in these conditions the tablets swelling properties, gel strength and diffusion of the bioadhesive polymer into the mucosa must be critical for the gastric bioadhesion.

CS is a cationic polysaccharide with a $\mathrm{p} K_{\mathrm{a}}$ value $\approx 6.5$, with a solubility and gel properties strongly influenced by $\mathrm{pH}$ medium. At $\mathrm{pH} 1.5, \mathrm{CS}$ is totally unionized, and tablets showed identical swelling behaviour independently on their CS content. In absence of polymer and mucin charges, tablet swelling rate and rheological properties of the gel formed conditioning the polymer mucoadhesion and so at $\mathrm{pH} 1.5$ all tablets have similar gastric bioadhesion.

At $\mathrm{pH} 4 a \approx 99 \%$ of the sialic acid in the gastric mucosa is ionized but only $1 \%$ of CS is in the cationic form. In this medium, gastric mucin is slightly more expanded than $\mathrm{pH} 1.5$, and CS tablets show differences in their swelling behaviour with CS content. Tablets with higher CS content shows a high initial swelling rate, due to a rapid water imbibition, continued for a significant erosion process. The presence of charges and the rapid initial swelling rate must favor the establishment of strong bioadhesive interactions given that $\mathrm{pH} 4 \mathrm{CS}$ tablets showed higher biodhesion that $\mathrm{pH}$ 1.5. Both work of adhesion and maximum detachment forces increase with CS content, especially in batch C. Nevertheless, the erosion process weakens the gel strength, which produces the failure in the bioadhesive interface and thus the detachment of the tablets. In samples with higher $\mathrm{CS}$ content at $\mathrm{pH} 4$, a failure in the gel formed in the interface of the tablet with gastric mucosa was observed. After bioadhesive assay adhered polymer was detected in the surface of the mucosa, it was confirmed that the breakage of the bioadhesive union was produced in the matrix gel, but not in gel-mucosa interaction layer. Therefore, mechanical properties of gel formed could be critical at this point.

Figure 5 shows the work of bioadhesion and maximum detachment forces of tablets elaborated with CS, HPMC and their mixtures. Incorporation of HPMC in tablets increased their gastric bioadhesive properties at $\mathrm{pH}$ 1.5. Tablets elaborated with HPMC (batch D) or mixtures HPMC/CS (batch E) showed the higher values of work of bioadhesion and maximum detachment forces at $\mathrm{pH} 1.5$. In these $\mathrm{pH}$ medium, tablets of $\mathrm{D}$ and $\mathrm{E}$ batches showed higher swelling rates than $\mathrm{A}$, B or C. Nevertheless, at $\mathrm{pH} 4$ medium, similar gastric bioadhesive work and maximum detachment forces were obtained for batch D elaborated with HPMC and for batch C containing $200 \mathrm{mg}$ of CS. Batch E, fabricated with the mixture of CS and HPMC (135-135 mg), showed lower values of bioadhesive work than $\mathrm{C}$ and $\mathrm{D}$ but higher maximum detachment forces. At difference of batch $\mathrm{C}$, no failure in the gel formed in the mucoadhesive interface was observed for tablets containing HPMC.

Dissolution Study The drug release from the tablets is conditioned by the swelling and erosion processes and the different solubilities of the excipients in the different media. Release profiles of ACV from tablets in acidic medium $\mathrm{pH} 1.5$ and $\mathrm{pH} 4$ were represented in Figs. 6 and 7, respectively.

Tablets with only CS (batches A, B and C) showed in $1.5 \mathrm{pH}$ medium a sustained release of ACV, and total drug 

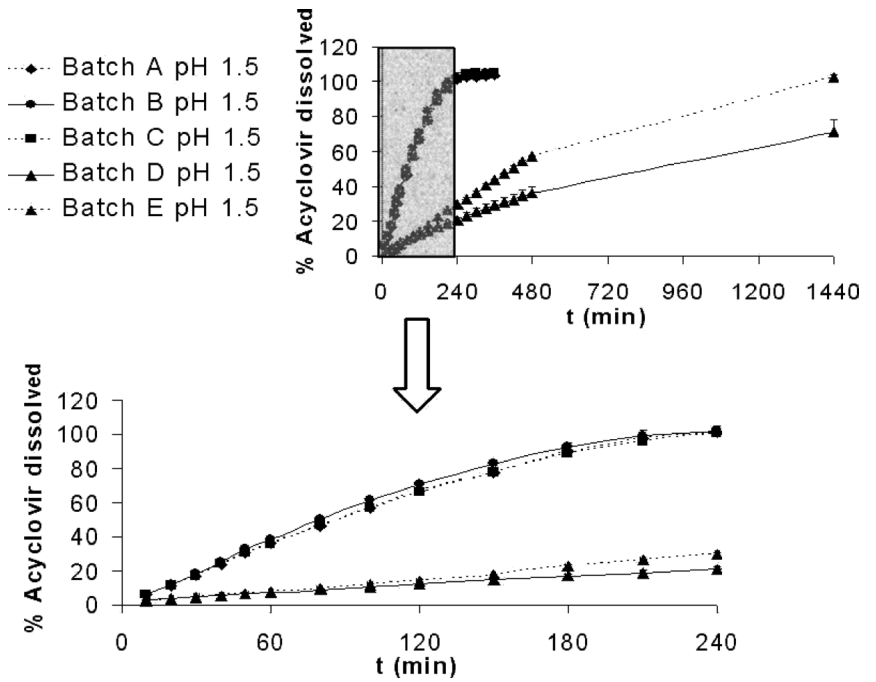

Fig. 6. Dissolution Profiles of Batches $\mathrm{A} \rightarrow \mathrm{E}$ in Acidic Medium of $\mathrm{pH}$ 1.5 (Mean Values \pm Standard Deviation, $n=3$ )

dissolution was obtained at $4 \mathrm{~h}$, even though no solid residue of tablet was detected. On the contrary, fast ACV release was obtained at $\mathrm{pH} 4$, and total drug dissolution was reached in $90 \mathrm{~min}$. There were clear differences between the replicates, which can be seen with the error bars (Fig. 7). This behaviour was attributed to the fact that, at $\mathrm{pH} 4$, ADP caused fast tablet disintegration and $\mathrm{ACV}$ dissolved from the large particles obtained.

Drug release from tablets with only HPMC (batch D) was slower, and only $20 \%$ and $14 \%$ of ACV were dissolved at $4 \mathrm{~h}$ in $\mathrm{pH} 1.5$ and $\mathrm{pH} 4$, respectively. Similar behaviour displayed tablets with both polysaccharides (batch E). In this case, only $30 \%$ (at $\mathrm{pH} \mathrm{1.5)} \mathrm{and} \mathrm{16 \%} \mathrm{(at} \mathrm{pH} 4$ ) of drug were released at $4 \mathrm{~h}$ of dissolution test. The explanation could be that HPMC behaviour (slow medium absorption and compact gel layer around the tablet) prevail over the possible CS action in batch E (imbibition and progressive dissolution), or ADP behaviour in batch $\mathrm{D}$ (slow dissolution at $\mathrm{pH} 1.5$ and disintegrate action at $\mathrm{pH}$ 4). Moreover, dissolution tests of batches $\mathrm{D}$ and $\mathrm{E}$ were prolonged for $24 \mathrm{~h}$ and at this time, ACV dissolution from batch $\mathrm{E}$ was $100 \%$ and $66 \%$ at $\mathrm{pH} 1.5$ and $\mathrm{pH} 4$, respectively, however, from batch $\mathrm{D}$ only $71 \%$ (at $\mathrm{pH} 1.5$ ) and $38 \%$ (at $\mathrm{pH}$ 4) of drug was released. Furthermore, tablets from batch D remained as compact monolithic structures after their stay in both dissolution media ( $\mathrm{pH} 1.5$ and 4) for $24 \mathrm{~h}$.

All dissolution data are in agreement with swelling be-
... Batch $\mathrm{A} \mathrm{pH} 4$

- Batch B pH 4

-. Batch $\mathrm{C} \mathrm{pH} 4$

$\rightarrow$ Batch D pH 4

- Batch $\mathrm{E} \mathrm{pH} 4$
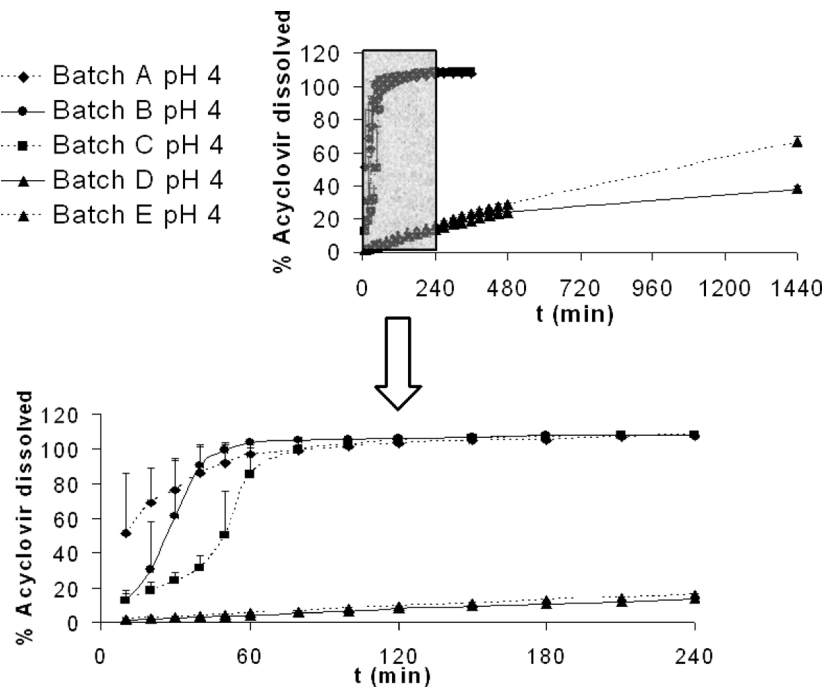

Fig. 7. Dissolution Profiles of Tablets $\mathrm{A} \rightarrow \mathrm{E}$ in Acidic Medium of $\mathrm{pH} 4$ (Mean Values \pm Standard Deviation, $n=3$ )

haviour because ACV release from all batches studied was conditioned by the behaviour of the polymer included in each tablet composition (imbibition and dissolution in the case of CS and slow imbibition and compact gel layer formation in the case of HPMC).

In order to determine the mechanism of drug release, mean data of ACV dissolved from the batches tested which have prolonged drug release: $\mathrm{D}$ and $\mathrm{E}$ in both media and $\mathrm{A}, \mathrm{B}$ and $\mathrm{C}$ in $\mathrm{pH} 1.5$, were subjected to release kinetic studies and its respective $R^{2}$ and costant values are given in Table 4.

The best fit with the highest correlation coefficient was shown in Hixson-Crowell for batches D and $\mathrm{E}$ and Korsmeyer and Peppas and Hopfenberg equation for A, B and C tablets as shown in Table 4. The release of the drug followed these kinetics and regression values indicated fair linearity in the data. The regression value in the cases $\mathrm{D}$ and $\mathrm{E}$ in both media are closer to unity in case Hixson-Crowell plot, and therefore, the release occurs in planes that are parallel to the drug surface because the tablet dimensions diminish proportionally (in such a manner that the initial geometrical form keeps constant the entire time). As less correlation was observed in the Higuchi plot, the drug release from these tablets was not diffusion controlled. The data indicated poor linearity, and was less than the previous values when it was plotted according to the first order and Higuchi equation.

The data drug release of the batches $\mathrm{D}$ and $\mathrm{E}$ were also

Table 4. Kinetic Parameters $\left(R^{2}\right.$ and Constants of First Order, Higuchi, Hixson-Crowell, Korsmeyer-Peppas and Hopfenberg Model) of Selected Acyclovir Tablets (C) with Controlled Release of Acyclovir (Mean Values, $n=3$ )

\begin{tabular}{cccccccccccc}
\hline \hline \multirow{2}{*}{ Batch } & \multicolumn{2}{c}{ First order kinetic } & \multicolumn{2}{c}{ Higuchi model } & \multicolumn{2}{c}{ Hixson-Crowell model } & \multicolumn{2}{c}{ Korsmeyer-Peppas model } & \multicolumn{2}{c}{ Hopfenberg model } \\
\cline { 2 - 13 } & \multicolumn{1}{c}{$K_{1}$} & $R^{2}$ & $K_{\mathrm{h}}$ & $R^{2}$ & $K s$ & $R^{2}$ & $a$ & $n$ & $R^{2}$ & $K_{1}$ & $R^{2}$ \\
\hline A pH 1.5 & 0.0122059 & 0.7821 & 0.0765 & 0.9790 & 0.0149 & 0.9746 & 0.0052626 & 1.0239 & 0.9983 & 0.0039 & 0.9934 \\
B pH 1.5 & 0.0119756 & 0.7660 & 0.0781 & 0.9751 & 0.0169 & 0.9704 & 0.0051988 & 1.0484 & 0.9981 & 0.0043 & 0.9949 \\
C pH 1.5 & 0.0119756 & 0.7835 & 0.0759 & 0.9795 & 0.0140 & 0.9876 & 0.0057346 & 1.0035 & 0.9978 & 0.0038 & 0.9979 \\
D pH 1.5 & 0.0048363 & 0.8668 & 0.0186 & 0.9825 & 0.0013 & 0.9991 & 0.0038967 & 0.7269 & 0.9923 & 0.0004 & 0.9985 \\
E pH 1.5 & 0.0062180 & 0.8583 & 0.0300 & 0.9645 & 0.0024 & 0.9958 & 0.0016823 & 0.9392 & 0.9943 & 0.0007 & 0.9977 \\
D pH 4 & 0.0052969 & 0.8537 & 0.0125 & 0.9817 & 0.0008 & 0.9983 & 0.0017701 & 0.7900 & 0.9933 & 0.0003 & 0.9978 \\
E pH 4 & 0.0050666 & 0.8646 & 0.0149 & 0.9794 & 0.0010 & 0.9988 & 0.0025293 & 0.7603 & 0.9937 & 0.0003 & 0.9984 \\
\hline
\end{tabular}


subjected to Korsmeyer-Peppas model in order to find $n$ value, which describes the drug release mechanism. This model is generally used to analyze the release of pharmaceutical polymeric dosage forms when the release mechanism is not well known or when more than one type of release phenomena could be involved. Both types of tablets exhibited $n$ values between 0.5 and 1 , indicating drug release was zero order controlled by an anomalous transport with non-Fickian diffusion.

The data obtained in $\mathrm{A}, \mathrm{B}$ and $\mathrm{C}$ tablets were also put in Korsmeyer-Peppas model. They fitted well with Korsmeyer-Peppas models as their $R^{2}$ values in the range of 0.9923-0.9978 with $n$ values in acidic medium of $\mathrm{pH} 1.5$ above or equal to 1 , therefore the drug release mechanism followed case II or super case II transport mechanism based on Korsmeyer-Peppas equation. The tablets with a high CS amount (batch $\mathrm{C}$ ) showed a $n$ value close to 1 corresponded to zero order release (case II transport), while A and B tablets with less proportions of CS presented a super case II transport, therefore, the mechanisms of drug release was non-Fickian diffusion. This indicates that drug release depends on swelling, relaxation and erosion of polymer with zero order release kinetics. All data release of the studied systems (batches $\mathrm{A} \rightarrow \mathrm{E}$ ) fitted to Hopfenberg model, which describe drug release from slabs, spheres and infinite cylinders displaying heterogeneous erosion, showed regression values indicating fair linearity in the data $\left(R^{2}>0.99\right)$. Thus, it could be assumed that the rate-limiting step of drug release is the erosion of the matrices itself and that time dependent diffusional resistances internal or external to the eroding matrices do not influence it.

The kinetic constant in Hopfenberg $\left(K_{1}\right)$ and KorsmeyerPeppas (a) models were not dependent of the amount of CS in the tablets $\mathrm{A}, \mathrm{B}$ and $\mathrm{C}$, however, these constant values were higher in the ACV/ADP/CS tablets than in the batches D and $\mathrm{E}$ in both models due to these last showed a more prolonged drug release.

\section{Conclusion}

Mucoadhesive CS and/or HPMC tablets for gastric drug delivery of ACV have been developed in order to improve the ACV oral bioavailability. Swelling, bioadhesive and dissolution studies in two acidic media $(\mathrm{pH} 1.5$ and 4) proved that HPMC and CS could be suitable polymers to obtain tablets which showed good mucoadhesion behaviour on gastric mucosa and ACV controlled release profiles. However, among all the studied batches, the tablets formulated only with CS would be the most suitable to improve ACV oral bioavailability, as the total amount of ACV was released into $4 \mathrm{~h}$, and no solid residue from tablets was detected due to the CS imbibition and erosion processes in $\mathrm{pH} 1.5$ medium. These successful results can consider themselves as the beginning for planning the in vivo evaluation in animal models.

Acknowledgments Roberto Ruiz Caro was a recipient of a predoctoral scholarship from the UCM (Spain) to do his $\mathrm{PhD}$. This research was supported by an UCM project (PR27/05-13878). Manuel Gago Guillán acknowledges the Isabel Barreto contract supported by the Xunta de Galicia (Spain). The authors would like to express their sincere gratitude to Margit Liander for the English check of the manuscript.

\section{References}

1) Illum L., Pharm. Res., 15, 1326-1331 (1998).

2) Skaugrud O., Drug Cosmet. Ind., 148, 24-29 (1991).

3) Ilango R., Jayakar B., Kavimani S., The East. Pharm., 41, 47-49 (1998).

4) Singla A. K., Chawla M., J. Pharm. Pharmacol., 53, 1047-1067 (2001).

5) Ubaidulla U., Khar R. K., Ahmad F. J., Sultana Y., Panda A. K., J. Pharm. Sci., 96, 3010-3023 (2007).

6) Lueßen H. L., Lehr C. M., Rentel C. O., Noach A. B. J., de Boer A. G., Verhoef J. C., Junginger H. E., J. Controlled Release, 29, 329-338 (1994).

7) Lueßen H. L., de Leeuw B. J., Langemeÿer M. W., de Boer A. (Bert) G., Verhoef J. C., Junginger H. E., Pharm. Res., 13, 16681672 (1996)

8) Lueßen H. L., Rentel C. O., Kotzé A. F., Lehr C. M., de Boer A. G., Verhoef J. C., Junginger H. E., J. Controlled Release, 45, 15-23 (1997).

9) Hassan E. E., Gallo J. M., Pharm. Res., 7, 491-495 (1990).

10) Narkar M., Sher P., Pawar A., AAPS PharmSciTech, 11, 267-277 (2010).

11) Tiwari S. B., Rajabi-Siahboomi A. R., Methods Mol. Biol., 437, 217-243 (2008)

12) Maggi L., Machiste E. O., Torre M. L., Conte U., Eur. J. Pharm. Biopharm., 48, 37-42 (1999).

13) Velasco M. V., Ford J. L., Rowe P., Rajabi-Siahboomi A. R., J. Controlled Release, 57, 75-85 (1999).

14) Varghas C. I., Ghaly E. S., Drug Dev. Ind. Pharm., 25, 1045-1050 (1999).

15) Sako K., Sawada T., Nakashima H., Yokohama S., Sonobe T., J. Controlled Release, 81, 165-172 (2002).

16) Bettini R., Catellani P. L., Santi P., Massimo G., Peppas N. A., Colombo P., J. Controlled Release, 70, 383-391 (2001).

17) Colombo P., Bettini R., Santi P., de Ascentiis A., Peppas N. A., J. Controlled Release, 39, 231-237 (1996).

18) Colombo P., Bettini R., Peppas N. A., J. Controlled Release, 61, 83-91 (1999).

19) Colombo P., Bettini R., Santi P., Peppas N. A., Pharm. Sci. Technol. Today, 3, 198-204 (2000).

20) Erlich K. S., West. J. Med., 166, 211-215 (1997).

21) Fujioka Y., Mizuno N., Morita E., Motozono H., Takahashi K., Yamanaka Y., Shinkuma D., J. Pharm. Pharmacol., 43, 465-469 (1991).

22) Meadows K. C., Dressman J. B., Pharm. Res., 7, 299-303 (1990).

23) Vergin H., Kikuta C., Mascher H., Metz R., Arzneimittelforschung, 45, 508-515 (1995).

24) de Miranda P., Krasny H. C., Page D. A., Elion G. B., Am. J. Med., 73 (1A), 31-35 (1982).

25) Attia I. A., El-Gizawy S. A., Fouda M. A., Donia A. M., AAPS PharmSciTech, 8, E106 (2007).

26) Peppas N. A., Sahlin J. J., Biomaterials, 17, 1553-1561 (1996).

27) Smart J. D., Adv. Drug Deliv. Rev., 57, 1556-1568 (2005).

28) Tao Y., Lu Y., Sun Y., Gu B., Lu W., Pan J., Int. J. Pharm., 378, 30-36 (2009).

29) Belgamwar V., Shah V., Surana S. J., Curr. Drug Deliv., 6, 113-121 (2009).

30) Dhaliwal S., Jain S., Singh H. P., Tiwary A. K., AAPS J., 10, 322330 (2008).

31) Liu H., Pan W., Ke P., Dong Y., Ji L., Drug Dev. Ind. Pharm., 36, 1098-1105 (2010).

32) Shadab, Ahuja A., Khar R. K., Baboota S., Chuttani K., Mishra A. K., Ali J., Drug Deliv., 18, 255-264 (2011).

33) Dressman J. B., Berardi R. R., Dermentzoglou L. C., Russell T. L., Schmaltz S. P., Barnett J. L., Jarvenpaa K. M., Pharm. Res., 7, 
756-761 (1990).

34) Russell T. L., Berardi R. R., Barnett J. L., Dermentzoglou L. C., Jarvenpaa K. M., Schmaltz S. P., Dressman J. B., Pharm. Res., 10, 187-196 (1993).

35) Lui C. Y., Amidon G. L., Berardi R. R., Fleisher D., Youngberg C., Dressman J. B., J. Pharm. Sci., 75, 271-274 (1986).

36) Arora S., Ali J., Ahuja A., Khar R. K., Baboota S., AAPS PharmSciTech, 6, E372-E390 (2005).

37) Haupt S., Zioni T., Gati I., Kleinstern J., Rubinstein A., Eur. J. Pharm. Sci., 28, 204-211 (2006).

38) Blanco-Fuente H., Anguiano-Igea S., Otero-Espinar F. J., Blanco-Mendez J., Int. J. Pharm., 142, 169-174 (1996).

39) Blanco-Fuente H., Vila-Dorrio B., Anguiano-Igea S., Otero-Espinar
F. J., Blanco-Mendez J., Int. J. Pharm., 138, 103-112 (1996).

40) Costa P., Sousa Lobo J. M., Eur. J. Pharm. Sci., 13, 123-133 (2001).

41) Higuchi T., J. Pharm. Sci., 52, 1145-1149 (1963).

42) Hixson A. W., Crowell J. H., Ind. Eng. Chem., 23, 923-931 (1931).

43) Korsmeyer R. W., Gurny R., Doelker E. M., Buri P., Peppas N. A., Int. J. Pharm., 15, 25-35 (1983).

44) Hopfenberg H. B., "Controlled Release Polymeric Formulations (ACS Symposium Series 33)," ed. by Paul D. R., Harris F. W., American Chemical Society, Washington D.C., 1976, pp. 26-31.

45) Ruiz-Caro R., Veiga M. D., Materials, 3, 5195-5211 (2010).

46) Mantle M., Allen A., Biochem. J., 195, 267-275 (1981).

47) Marshall T., Allen A., Biochem. J., 173, 569-578 (1978). 